\title{
The Microbiome and Asthma
}

\author{
Yvonne J. Huang ${ }^{1}$ and Homer A. Boushey ${ }^{1}$ \\ ${ }^{1}$ Division of Pulmonary/Critical Care/Sleep and Allergy/Immunology, University of California San Francisco, San Francisco, California
}

\section{Abstract}

That the subglottic airways are not sterile, as was once believed, but are populated by a distinct "bronchial microbiome," is now accepted. Also accepted is the concept that asthma is associated with differences in the composition of this microbiome. What is not clear is whether the differences in microbial community composition themselves mediate pathologic changes in the airways or whether they reflect differences in systemic immune function driven by differences in the development of the gastrointestinal microbiome in early life, when the immune system is most malleable. Recognition of the probable existence of a "common mucosal immune system" allowed synthesis of these apparently opposing ideas into a single conceptual model. Gastrointestinal microbiome-driven differences in systemic immune function predispose to sensitization to allergens deposited on mucosal surfaces, whereas possibly similar, but not identical, differences in immune function predispose to less effective responses to microbial infection of the airways, resulting in persistence of the inflammation underlying the structural and functional abnormalities of asthma. In this model, allergic sensitization and asthma are thus seen as commonly overlapping but not necessarily coincident consequences of abnormalities in microbial colonization, development of immune function, and encounter with agents infecting the respiratory tract.

Keywords: asthma; bronchial microbiome; gastrointestinal microbiome; respiratory infection

(Received in original form June 20, 2013; accepted in final form September 1, 2013)

Correspondence and requests for reprints should be addressed to Homer A. Boushey, M.D., 505 Parnassus Avenue, M1292, Box 0130, San Francisco, CA 94143-0130. E-mail: hab2@itsa.ucsf.edu

Ann Am Thorac Soc Vol 11, Supplement 1, pp S48-S51, Jan 2014

Copyright (C) 2014 by the American Thoracic Society

DOI: 10.1513/AnnalsATS.201306-187MG

Internet address: www.atsjournals.org

Interest in the possible role of microbes in the pathogenesis, clinical expression, and progression of asthma first focused on infection with a single organism potentially amenable to antibiotic treatment. With the development of culture-independent tools for detecting microbes by genetic sequence and with the resulting description of a vastly greater number and variety of microbes than had previously been known to be present in different anatomical sites, especially on mucosal surfaces, interest shifted to the function of complex microbial communities in health and disease. Whether a bacterial community is actually present in the tracheobronchial tree, and, if so, whether it differs in subjects with asthma and healthy subjects, has been directly investigated, and both suppositions have been confirmed: the bronchial airways indeed appear to be colonized by a microbial community distinct from that in the oropharynx, and this bronchial microbiome differs in people in good health versus those with asthma, chronic obstructive pulmonary disease, or cystic fibrosis.

Studies of samples obtained directly from the bronchial airways have suggested associations between differences in the microbial communities with differences in bronchial health. Other studies have also suggested associations between differences in the microbial communities found in environmental samples with differences in the risk of developing airway disease, especially of asthma. The evidence from these studies is indirect and has shown the strongest associations with the development of asthma to be with features of the microbial environment in the first 3 years of life. These findings broadly fit the conceptual model of the "hygiene hypothesis," in which environmental exposures are believed to affect composition and function of the gut microbiome, which in turn shapes the pattern of development of systemic immune function and the risk of diseases of immune dysregulation and chronic inflammation.

These observations and interpretations have been synthesized into a conceptual model of asthma's pathogenesis. At the heart of the model are two ideas. One is that differences in microbial colonization of the gastrointestinal tract in infancy result in microbially driven differences in the rate and pattern of development of systemic immune function, predisposing to allergic sensitization. The other is that there is "crosstalk" between mucosal compartments, so that microbially driven differences in mucosal immune function at one site may be shared across mucosal surfaces at other sites, in what has been described as "the (mostly) Common 
Mucosal Immune System" (emphasis added) (1). In this conceptual model, these differences in systemic and local immune function are conceived as altering the response to infection, whether bacterial, viral, or fungal, at the mucosal surface of the airways in a way that results in the characteristic inflammation, mucoid metaplasia, and bronchial hyperreactivity of asthma.

\section{History: Reasons for Interest in Airway Microbiota in Asthma}

The idea that infection might underlie some forms of asthma was fostered by reports in the 1950s that treatment with trimethyl oleandomycin, a macrolide antibiotic, was effective in patients with "chronic infectious asthma" (2), the diagnosis then assigned to patients with asthma whose sputum increased in volume and purulence in association with asthma exacerbations. The later demonstration of Mycoplasma pneumoniae or Chlamydophila pneumoniae by polymerase chain reaction (PCR) of bronchial biopsies in a much higher proportion of patients with asthma than healthy patients seemed to confirm the idea of an infectious component to asthma's pathogenesis (3), as did the clinical improvements brought about by prolonged clarithromycin treatment of PCR-positive subjects $(4,5)$.

The findings from the Copenhagen Birth Cohort Study suggested that different bacteria were importantly involved. In this study of more than 600 infants, culture of Moraxella catarrhalis, Haemophilus influenzae, or Streptococcus pneumoniae from the oropharynx at 1 month of age was associated with a significant increase in the odds ratio for childhood asthma (6). The same bacteria have been found to be as strongly associated with asthma exacerbations in children as are respiratory viruses (7).

\section{"Disordered" Bronchial Microbiota in Asthma}

Culture-independent methods for identifying bacteria by amplifying and sequencing regions of the gene for $16 \mathrm{~S}$ ribosomal RNA conserved across bacteria and archaea have been applied to samples obtained by bronchoscopy of volunteers with asthma and healthy volunteers in two studies. The study by Hilty and colleagues (8) of respiratory samples, including bronchial brushings from 11 adults with asthma, 5 with COPD, and 8 healthy control subjects, identified bacteria by a traditional $16 \mathrm{~S}$ rRNA clone library-serial sequencing approach (34-63 sequenced clones/subject). They showed that compared with healthy control subjects, subjects with asthma had significantly more pathogenic Proteobacteria, such as Haemophilus species, but fewer Bacteroidetes, especially Prevotella species (8). Huang and colleagues more comprehensively analyzed $16 \mathrm{~S}$ rRNA sequences using a massively parallel microarray approach (PhyloChip) in their larger study of protected bronchial brush samples from 42 subjects with asthma and 5 healthy subjects (9). As in Hilty and colleagues' study (8), all of the subjects with asthma were taking an inhaled corticosteroid. Bacterial communities were identified in both asthmatic and healthy groups, but the bacterial burden was significantly greater in patients with asthma than in healthy control subjects and, among patients with asthma, in those with greater bronchial reactivity to methacholine. In addition, patients with asthma whose bronchial reactivity improved after 6 weeks of clarithromycin treatment had higher baseline bacterial diversity (9). The bacterial populations identified were polymicrobial and in the patients with asthma showed a trend toward greater abundance of Proteobacteria (e.g., Pseudomonas, Haemophilus, and many other species). In addition, greater bronchial reactivity correlated with greater relative abundance of particular bacterial community members with known functional characteristics of possible relevance to the pathophysiology of asthma. As examples, these included bacterial species capable of expression of nitric oxide reductase, production of sphingolipids, and metabolism of steroid compounds, or those that demonstrate sensitivity to macrolide antibiotics, which have been used with variable degrees of success in the treatment of chronic asthma (10).

These findings in patients with asthma taking an inhaled corticosteroid were importantly extended by Marri and colleagues in their study of induced sputum samples from 10 healthy young adults and 10 young adults with asthma followed from birth in the Tucson Children's Respiratory Study (11). Because the patients with asthma were not taking treatment with an inhaled corticosteroid at the time of the study, the finding that their sputum samples contained greater abundance of Proteobacteria, again especially Haemophilus species, and lower abundance of Firmicutes indicates that the difference in bronchial microbiota is a feature of asthma itself and not necessarily of the immunomodulatory corticosteroid treatment taken for it.

\section{Mechanisms of Protection or Harm from Airway Microbes}

It is not known whether the "disordered" bronchial microbiome described by these studies itself mediates the disturbances of asthma or simply reflects an underlying disorder in immune function permitting its development. This has been little studied, but in an attempt to elucidate the question, Følsgaard and colleagues examined the association between airway colonization with pathogenic bacteria and immune signature of upper airway in neonates by measuring cytokines in nasal samples of neonates in the Copenhagen Birth Cohort study (12). They found colonization with $H$. influenzae or $M$. catarrhalis to be associated with increases suggesting a mixed Th1, Th2, Th17 response; $S$. pneumoniae with no increases; and Staphylococcus aureus with Th1 and strong Th17 responses. They speculated that the less-effective immune response to $H$. influenzae, $M$. catarrhalis, and $S$. pneumoniae results in persistence of the bacteria, promoting chronic airway inflammation, whereas the more efficient elimination of $S$. aureus enables the bronchial mucosa to return to a normal, quiescent state.

Conceiving of the bronchial microbiome as entirely bacterial would, of course, be incomplete, but methods for highthroughput description of fungi and viruses in airway samples are not yet widely available. A recent comparison of the fungi identified by 454 sequencing of induced sputum samples pooled from subjects with asthma versus healthy subjects found that 90 fungal species were more abundant in subjects with asthma and 46 more abundant in the control subjects (13). That fungi may alter airway function through mechanisms 
other than acting as a persistent allergenic stimulus has been excitingly suggested in a preliminary report of Aspergillus's expression of a lipoxygenase with substantial homology to human 5 lipoxygenase (14).

Another way by which "disorder" of the microbiota normally present in a host niche might result in disease is through the failure to inhibit the outgrowth of pathogens. Examples of this are the antibiotic-associated diseases due to outgrowth of microbes present in health, such as oral and vaginal candidiasis and Clostridium difficile colitis, and the newly demonstrated role of Corynebacterium tuberculostericum in chronic rhinosinusitis (15). This loss of the protective function of the microbiome normally present, enabling outgrowth of an (hypothesized) asthmacausing pathogen or group of pathogens, could conceivably account for the association between antibiotic use in infancy and later development of childhood asthma (16).

\section{Microbial Associations with Growing up with Dogs}

The well-documented protective effects of breastfeeding, growing up in close contact with stables, and consuming farm milk are reviewed elsewhere in these proceedings (see von Mutius, pp. S13-S15) (17). That close contact with pet dogs in the first 3 years of life is similarly protective against the development of allergic sensitization and asthma has also been established (18). Reasoning that (1) environmental exposures in early life shape risk for later development of allergies and asthma, (2) gut microbiota shape immune function at mucosal surfaces, (3) ingestion of microbes and of microbial nutrients shape development of gut microbiome, and (4) infants ingest house dust, we hypothesized that dust from households with dogs must contain microbes protective against allergy and asthma. That the presence of dogs affects the microbiota of house dust was forcefully illustrated in a recent study of multiple sites in 40 North Carolina homes, so much so that the authors concluded that "dogs appear to be the main contributor to differences in microbial diversity across homes" (19). That having a dog in the home increases the sharing of microbes among family members has also been illustrated in a study of microbiota in feces and on tongue, forehead, and palms in 60 families with dogs, children, both, or neither (20). Members of same household were found to share more microbiota with each other than with members of other households, and the presence of a dog in the household was found to increase the sharing of microbiota even more than the presence of a child. Our own investigation of the hypothesis showed greater richness, evenness, and diversity of the microbiota in house dust from dog-keeping versus petfree homes (21) and that this appeared to be true as well of stool samples from 6-month-old children living in such households.

\section{Role of Viral Respiratory Infection}

Several prospective birth cohort studies have now shown that infants who develop severe illness from a viral respiratory infection, especially if due to human rhinovirus, in the first year of life, have a much greater likelihood of being diagnosed with asthma at age 6 years (22). The development of a severe illness from human rhinovirus could be regarded as simply an "unmasking" of an asthmatic phenotype, but for an epidemiologic study's clear demonstration that children born 120 days before the peak week of the winter virus season have a significantly increased odds ratio of being treated for asthma 5 years later (23).

An alternative explanation is that the risk of asthma is shaped by the maturity of antiviral immune defense at the time of infection with an "asthmagenic" strain of virus. Antiviral immune defense has clearly been shown to be regulated by gut microbiota in animal models $(24,25)$, so it seems entirely possible that an infant's gut microbiome's effects on the response to viral infection are more important than its effects on allergic sensitization for asthma prevention. Indeed, some well-designed, double-blind, placebo-controlled studies of children have reported that feeding bacteria or bacterial products to children reduces the severity of their response to community-acquired respiratory tract infections $(26,27)$. Linking the findings of these studies to possible mechanisms of protection against asthma from growing up with dogs is a recent report of relationships among perinatal pet exposure, fecal microbiota, and wheezy bronchitis (28). Examined were 129 infants from a larger study of 256 pregnant women treated daily with probiotics (Bifidobacterium lactis and Lactobacillus rhamnosus) and dietary counseling, dietary counseling alone, or neither. Stool samples from the infants were examined by quantitative PCR for five species of Bifidobacteria, four of Clostridia, S. aureus, and Akkermansia muciniphila. Among the pet-exposed children, none of 30 had an episode of wheezy bronchitis by age 2 years, and $8(27 \%)$ had a positive skin test, whereas 15 (15\%) of the 99 children not exposed to household pets had an episode of wheezy bronchitis, and 42 (42\%) were atopic by skin testing. Although the examination of stool microbiota was far from comprehensive, it did show significant differences in the abundance of two species of bifidobacteria: Bifidobacterium longus was more abundant in the samples from pet-exposed infants, and Bifidobacterium brevis was more abundant in nonexposed infants. This finding serves as a reminder that important functional contributions by particular microbiota can differ at the species level (or even strain level). However, depending on the bacterial group, it can be difficult to discern specific species or strains of bacteria by the $16 \mathrm{~S}$ rRNA-based methods that have largely been used in studies to date of the microbiome.

\section{Asthma as a Preventable Disease-Elements of the Argument}

The conceptual model synthesized from these theories and findings can be outlined as follows:

- Some strains of common viruses play a role in the pathogenesis of asthma and other allergic diseases ("asthmagenic" viruses)

- The consequence of infection with these viruses is a function of the effectiveness of antiviral immune function at the time of infection.

- Antiviral immune function increases in efficacy in infancy.

- The rate of development of antiviral immune function is influenced by composition and function of the gut microbiome.

- Gut microbiome is modifiable by feeding and possibly by environmental exposure. 
This model is testable, holds great promise, and may be generalizable to chronic diseases other than asthma, including those affecting systems beyond those of the lungs and airways. Key to this model is recognition that the changes in the gut microbiota of human infants raised in wealthy "western" cultures may not themselves mediate disease but instead affect the susceptibility to infection with pathogens responsible-perhaps after long periods of latency - for chronic diseases appearing in childhood or even later in life.

\section{Future Directions}

The field of lung microbiome investigation has advanced rapidly.
Studies of the environmental, gastrointestinal, and respiratory microbiomes have generated new insights and novel hypotheses on potential causal relationships between the microbiome and asthma. Much remains to be studied regarding relationships between the microbiome and features of asthma. For example, it is well recognized that asthma is a heterogeneous disease, as has been described by a number of clinical and inflammatory characteristics. Could the microbiome play a greater role in the development of certain asthma phenotypes (or endotypes) than others? If so, knowing this would not only contribute to our understanding of asthma heterogeneity but also inform deliberations on future patient-tailored therapies, including any microbiometargeted treatment strategies that are developed. Tightly coupled with the need for more "characterization" studies is integrating investigation of the consequences of observed relationships between the microbiota and asthma, including functions expressed by the microbiota and the host. This will be no small challenge and will require thoughtful investigative strategies and likely multifaceted approaches.

Author disclosures are available with the text of this article at www.atsjournals.org.

\section{References}

1 Lawson LB, Norton EB, Clements JD. Defending the mucosa: adjuvant and carrier formulations for mucosal immunity. Curr Opin Immunol 2011;23:414-420.

2 Kaplan MA, Goldin M. The use of triacetyloleandomycin in chronic infectious asthma. Antibiot Annu 1958-1959;6:273-276.

3 Kraft M, Cassell GH, Henson JE, Watson H, Williamson J, Marmion BP, Gaydos CA, Martin RJ. Detection of Mycoplasma pneumoniae in the airways of adults with chronic asthma. Am J Respir Crit Care Med 1998;158:998-1001.

4 Kraft M, Cassell GH, Pak J, Martin RJ. Mycoplasma pneumoniae and Chlamydia pneumoniae in asthma: effect of clarithromycin. Chest 2002;121:1782-1788.

5 Good JT Jr, Kolakowski CA, Groshong SD, Murphy JR, Martin RJ. Refractory asthma: importance of bronchoscopy to identify phenotypes and direct therapy. Chest 2012;141:599-606.

6 Bisgaard H, Hermansen MN, Buchvald F, Loland L, Halkjaer LB, Bønnelykke K, Brasholt M, Heltberg A, Vissing NH, Thorsen SV, et al. Childhood asthma after bacterial colonization of the airway in neonates. N Engl J Med 2007;357:1487-1495.

7 Bisgaard H, Hermansen MN, Bønnelykke K, Stokholm J, Baty F, Skytt NL, Aniscenko J, Kebadze T, Johnston SL. Association of bacteria and viruses with wheezy episodes in young children: prospective birth cohort study. BMJ 2010;341:c4978.

8 Hilty M, Burke C, Pedro H, Cardenas P, Bush A, Bossley C, Davies J, Ervine A, Poulter L, Pachter L, et al. Disordered microbial communities in asthmatic airways. PLoS ONE 2010;5:e8578.

9 Huang YJ, Nelson CE, Brodie EL, Desantis TZ, Baek MS, Liu J, Woyke $\mathrm{T}$, Allgaier M, Bristow J, Wiener-Kronish JP, et al. Airway microbiota and bronchial hyperresponsiveness in patients with suboptimally controlled asthma. J Allergy Clin Immunol 2011;127:372-381.e1-3.

10 Good JT Jr, Rollins DR, Martin RJ. Macrolides in the treatment of asthma. Curr Opin Pulm Med 2012;18:76-84.

11 Marri PR, Stern DA, Wright AL, Billheimer D, Martinez FD. Asthmaassociated differences in microbial composition of induced sputum. J Allergy Clin Immunol 2013;131:346-352.e1-3.

12 Følsgaard NV, Schjørring S, Chawes BL, Rasmussen MA, Krogfelt KA, Brix S, Bisgaard $\mathrm{H}$. Pathogenic bacteria colonizing the airways in asymptomatic neonates stimulates topical inflammatory mediator release. Am J Respir Crit Care Med 2013;187:589-595.

13 van Woerden HC, Gregory C, Brown R, Marchesi JR, Hoogendoorn B, Matthews IP. Differences in fungi present in induced sputum samples from asthma patients and non-atopic controls: a community based case control study. BMC Infect Dis 2013;13:69.

14 Fischer GJ, Affeldt K, Berthier E, Keller NP. Fungal lipoxygenases: a novel instigator of asthma? 27th Fungal Genentics Conference,
Asilomar Conference Center. March 12-17, 2013, Asilomar Conference Center, Pacific Grove, CA.

15 Abreu NA, Nagalingam NA, Song Y, Roediger FC, Pletcher SD, Goldberg AN, Lynch SV. Sinus microbiome diversity depletion and Corynebacterium tuberculostearicum enrichment mediates rhinosinusitis. Sci Trans/ Med 2012;4:151ra124.

16 Marra F, Marra CA, Richardson K, Lynd LD, Kozyrskyj A, Patrick DM, Bowie WR, Fitzgerald JM. Antibiotic use in children is associated with increased risk of asthma. Pediatrics 2009;123:1003-1010.

17 von Mutius E. Environmental microorganisms and lung health. Ann Am Thorac Soc 2014;11:S13-S15.

18 Ownby DR, Johnson CC, Peterson EL. Exposure to dogs and cats in the first year of life and risk of allergic sensitization at 6 to 7 years of age. JAMA 2002;288:963-972.

19 Dunn RR, Fierer N, Henley JB, Leff JW, Menninger HL. Home life: factors structuring the bacterial diversity found within and between homes. PLOS ONE 2013;8:e64133.

20 Song SJ, Lauber C, Costello EK, Lozupone CA, Humphrey G, BergLyons D, Caporaso JG, Knights D, Clemente JC, Nakielny S, et al. Cohabiting family members share microbiota with one another and with their dogs. Elife 2013;2:e00458.

21 Fujimura KE, Johnson CC, Ownby DR, Cox MJ, Brodie EL, Havstad SL, Zoratti EM, Woodcroft KJ, Bobbitt KR, Wegienka G, et al. Man's best friend? The effect of pet ownership on house dust microbial communities. J Allergy Clin Immunol 2010;126:410-412.e1-3.

22 Jackson DJ, Lemanske RF Jr. The role of respiratory virus infections in childhood asthma inception. Immunol Allergy Clin North Am 2010; 30:513-522, vi.

23 Wu P, Dupont WD, Griffin MR, Carroll KN, Mitchel EF, Gebretsadik T, Hartert TV. Evidence of a causal role of winter virus infection during infancy in early childhood asthma. Am J Respir Crit Care Med 2008;178:1123-1129.

24 Yasui H, Kiyoshima J, Hori T. Reduction of influenza virus titer and protection against influenza virus infection in infant mice fed Lactobacillus casei Shirota. Clin Diagn Lab Immunol 2004;11:675-679.

25 Ichinohe T, Pang IK, Kumamoto Y, Peaper DR, Ho JH, Murray TS, Iwasaki A. Microbiota regulates immune defense against respiratory tract influenza A virus infection. Proc Natl Acad Sci USA 2011;108:5354-5359.

26 Razi CH, Harmancı K, Abacı A, Özdemir O, Hızıı S, Renda R, Keskin F. The immunostimulant OM-85 BV prevents wheezing attacks in preschool children. J Allergy Clin Immunol 2010;126:763-769.

27 Leyer GJ, Li S, Mubasher ME, Reifer C, Ouwehand AC. Probiotic effects on cold and influenza-like symptom incidence and duration in children. Pediatrics 2009;124:e172-e179.

28 Nermes M, Niinivirta K, Nylund L, Laitinen K, Matomäki J, Salminen S, Isolauri E. Perinatal pet exposure, faecal microbiota, and wheezy bronchitis: is there a connection? ISRN Allergy 2013;2013: 827934. 